\title{
Effects of drug pharmacokinetic/pharmacodynamic properties, characteristics of medication use, and relevant pharmacological interventions on fall risk in elderly patients
}

\author{
This article was published in the following Dove Press journal: \\ Therapeutics and Clinical Risk Management \\ 13 June 2014 \\ Number of times this article has been viewed
}

\author{
Ying Chen' \\ Ling-Ling Zhu \\ Quan Zhou ${ }^{3}$ \\ 'Liaison Office of Geriatric \\ VIP Patients, ${ }^{2}$ First Geriatric \\ VIP Ward, Division of Nursing, \\ ${ }^{3}$ Department of Pharmacy, \\ Second Affiliated Hospital, \\ School of Medicine, Zhejiang \\ University, Hangzhou, People's \\ Republic of China
}

Correspondence: Quan Zhou Department of Pharmacy, Second Affiliated Hospital, School of Medicine, Zhejiang University, 88 Jiefang Rd, Shangcheng, Hangzhou, Zhejiang 310009 , People's Republic of China

Tel +8657 I 87783690

Fax +86 57| 87022776

Email zhouquan142602@zju.edu.cn
Background: Falls among the elderly are an issue internationally and a public health problem that brings substantial economic and quality-of-life burdens to individuals and society. Falls prevention is an important measure of nursing quality and patient safety. Numerous studies have evaluated the association of medication use with fall risk in elderly patients. However, an upto-date review has not been available to summarize the multifaceted pharmaceutical concerns in the prevention of medication-related falls.

Materials and methods: Relevant literature was identified by performing searches in PubMed, Web of Science, and the Cochrane Library, covering the period until February 2014. We included studies that described an association between medications and falls, and effects of drug pharmacokinetic/pharmacodynamic properties, characteristics of medication use, and pharmacological interventions on fall risk in elderly patients. The full text of each included article was critically reviewed, and data interpretation was performed.

Results: Fall-risk-increasing drugs (FRIDs) include central nervous system-acting agents, cough preparations, nonsteroidal anti-inflammatory drugs, anti-Alzheimer's agents, antiplatelet agents, calcium antagonists, diuretics, $\alpha$-blockers, digoxin, hypoglycemic drugs, neurotoxic chemotherapeutic agents, nasal preparations, and antiglaucoma ophthalmic preparations. The degree of medication-related fall risk was dependent on one or some of the following factors: drug pharmacokinetic/pharmacodynamic properties (eg, elimination half-life, metabolic pathway, genetic polymorphism, risk rating of medications despite belonging to the same therapeutic class) and/or characteristics of medication use (eg, number of medications and drug-drug interactions, dose strength, duration of medication use and time since stopping, medication change, prescribing appropriateness, and medication adherence). Pharmacological interventions, including withdrawal of FRIDs, pharmacist-conducted clinical medication review, and computerized drug alerts, were effective in reducing fall risk.

Conclusion: Based on the literature review, clear practical recommendations for clinicians to prevent falls in the elderly included making a list of FRIDs, establishing a computerized alert system for when to e-prescribe FRIDs, seeking an alternative drug with lower fall risk, withdrawing FRIDs if clinically indicated, taking pertinent cautions when the use of FRIDs cannot be avoidable, paying attention to prescribing appropriateness, simplifying the medication regimen, strengthening pharmacist-conducted clinical medication review, ensuring the label of each FRID dispensed contains a corresponding warning sign, being careful when medication change occurs, enhancing medication adherence, and mandating for periodic reassessment of potential risk associated with the patient's medication regimen. Further studies should be conducted in this area, such as investigating whether medication reconciliation and improving medication adherence could decrease the rate of falls. 
Keywords: falls, geriatrics, medication, medication adherence, prescribing, risk, safe medication use

\section{Introduction}

Falls among the elderly are an issue internationally and a public health problem that brings substantial economic and quality-of-life burdens to individuals and society. About $28 \%-35 \%$ of people aged 65 years and over fall each year, whereas the rate of falls in those over 70 years of age increases to $32 \%-42 \%$. ${ }^{1}$ The rate of hospital admissions due to falls for people at aged 60 years and older in Australia, Canada, and the UK ranges from 1.6 to 3.0 per 10,000 population. ${ }^{1}$ Inpatient fall rates range from 1.7 to 25 falls per 1,000 patient days, depending on the care area. ${ }^{2}$

The Joint Commission, formerly known as the Joint Commission on Accreditation of Healthcare Organizations, sets international patient-safety goals to promote specific improvements in patient safety. The sixth goal is to reduce the risk of patient harm resulting from falls. The Joint Commission mandates to assess and periodically reassess each patient's risk for falling, including the potential risk associated with the patient's medication regimen, and take action to decrease or eliminate any identified risks. ${ }^{3}$

The use of some drugs may have a significant contribution to the occurrence of falls, due to their accessory side effects (such as sedation, dizziness, postural disturbances, altered gait and balance, or impaired cognition). These drugs are usually called fall-risk-increasing drugs (FRIDs). ${ }^{4}$

Numerous studies have evaluated the association of medication use with the risk of falling in elderly patients. Besides typical FRIDs such as central nervous system (CNS)-acting agents (benzodiazepines, sedatives, hypnotics, antidepressants, and antipsychotic drugs), in recent years relatively new classes (eg, anti-Alzheimer's agents, neurotoxic chemotherapeutic agents, and nasal preparations) were identified as having significant association with falls. ${ }^{5-7}$ Meanwhile, results of several sporadic cohort studies showed that the degree of medication-related fall risk was dependent on one or some of the following factors: drug pharmacokinetic and pharmacodynamic (PK/PD) properties (eg, elimination half-life, metabolic pathway, genetic polymorphism, risk rating of medications despite belonging to the same therapeutic class), and/or characteristics of medication use (eg, number of medications and drug-drug interactions [DDIs], dose strength, duration of medication use and time since stopping, medication change, prescribing appropriateness, and medication adherence). However, an up-to-date review has not been available to summarize such valuable information. Also, several randomized controlled trials have been designed to prevent falls through enhanced pharmaceutical care, with the aim of overcoming disadvantageous factors contributing to medication-related fall risk. The objective of this review is to introduce the concept of safe medication use to both patients and clinicians by presenting multifaceted pharmaceutical concerns in the prevention of medication-related falls among patients in all settings (community dwelling, nursing home, and hospital).

\section{Materials and methods}

\section{Search strategy}

Potentially relevant literature until February 2014 was identified by performing searches in the following databases using the search string "falls and medication and elderly" and an English-language filter: 1) PubMed (additional filter: aged 65+ years); 2) Web of Sciences (additional filter: proceedings paper, meeting abstract, and correction were excluded); and 3) the Cochrane Library.

\section{Selection criteria}

Two reviewers (YC and LLZ) independently searched the literature and screened the relevant studies. The numbers of papers identified in the three databases were 750, 803, and 407, respectively. After excluding duplicated literatures, 757 papers received further assessment. Studies were considered if they revealed the association between medications and falls, and studies describing the effects of drug's PK/PD properties, characteristics of medication use, or pharmacological interventions on fall risk in elderly patients. Each reviewer was blinded to the other reviewer in the process of data extraction. In the case of disagreement between the two reviewers, a third reviewer $(\mathrm{QZ})$ was consulted. Sixty-nine papers were finally included based on the inclusion/exclusion criteria. Valuable information was summarized by interpretation of the data after critically reading the full text of each paper.

\section{Results}

\section{Fall-risk-increasing drugs}

FRIDs are summarized in Table 1. These drugs, especially in the elderly, need to be reviewed regularly. Pharmacists should provide appropriate drug information related to the risk of falling to both patients and medical staff members. 
Table I Fall-risk-increasing drugs in the literature

\begin{tabular}{|c|c|}
\hline Category & Risk description and clinical management \\
\hline CNS-acting agents & $\begin{array}{l}\text { Retrospective case-control studies showed strong evidence of an association between substantially increased risk of } \\
\text { falls and drugs from this therapy group. Patients receiving CNS-acting agents were ten times more likely to have fall } \\
\text { risk (OR } 9.90 ; 95 \% \mathrm{Cl} \text { I.6-60.63). }{ }^{8-11} \text { Inpatient falls were significantly associated with patients taking anti-Parkinson's } \\
\text { medication (OR about 4-5). } .^{10,12}\end{array}$ \\
\hline Cough preparations & $\begin{array}{l}\text { Cough preparations are known to cause sedation and more frequent visits to the toilet, due to worsening bladder- } \\
\text { outlet obstruction in elderly men. More fallers were taking cough mixture compared with nonfallers }(5.7 \% \text { versus } \\
\text { I.0\%, } P=0.00 \mathrm{I}) \text {, indicating hospitalized patients on cough preparations were more likely to fall. }{ }^{13}\end{array}$ \\
\hline NSAIDs & $\begin{array}{l}\text { NSAID use was a significant predictor of falls in hospitalized elderly patients and was associated with a tenfold } \\
\text { increase in the likelihood of falling (OR } 10.02,95 \% \mathrm{Cl} 2.6-38.58 ; P=0.00 \mathrm{I}) . .^{14}\end{array}$ \\
\hline Anti-Alzheimer's agents & $\begin{array}{l}\text { The use of Alzheimer's medication (eg, donepezil, rivastigmine, galantamine, memantine) was associated with fall } \\
\text { risk (HR I.63, } 95 \% \mathrm{Cl} \text { I.24-2.14; } P=0.0005) \text {. Additional pharmacovigilance of the association between falls and } \\
\text { Alzheimer's medication use is warranted. }{ }^{6}\end{array}$ \\
\hline Antiplatelet agents & $\begin{array}{l}\text { A study in elderly Asian patients in an acute care setting, including } 298 \text { fallers and } 298 \text { matched nonfallers, indicated } \\
\text { that hospitalized patients on antiplatelet agents were more likely to fall. More fallers were taking antiplatelet agents } \\
\text { compared with nonfallers }(I 5.9 \% \text { versus } I .3 \%, P<0.00 I) .{ }^{13}\end{array}$ \\
\hline Calcium antagonists & The probability of falls increased when patients used calcium antagonists (aOR $2.45,95 \% \mathrm{Cl} I .16-4.74 ; P=0.02) . "$ \\
\hline Diuretics & $\begin{array}{l}\text { Nursing home residents are at an increased risk of falls on the day following a new prescription or increased dose } \\
\text { of a loop diuretic drug (OR } 2.46,95 \% \mathrm{Cl} \text { I.02-5.92). It was estimated that for every } 27 \mathrm{I} \text { loop diuretic drug changes, } \\
\text { one excess fall occurred. Extra precautions should be taken immediately following a loop diuretic drug change in an } \\
\text { effort to prevent falls. }{ }^{15} \mathrm{~A} \text { case-control study showed that current prescribing of thiazides was associated with an } \\
\text { increased risk of falling, and that this was strongest in the } 3 \text { weeks following the first prescription (OR } 4.28,95 \% \mathrm{Cl} \\
\text { I.19-15.42). }{ }^{16}\end{array}$ \\
\hline$\alpha$-blockers & $\begin{array}{l}\text { Current use of standard-formulation } \alpha \text {-blockers (prazosin, doxazosin, terazosin, alfuzosin, and tamsulosin) was } \\
\text { associated with an increased risk of hip/femur fracture, commonly due to falls (aOR I.9, } 95 \% \mathrm{Cl} \text { I.I-3.0). The effect } \\
\text { was particularly strong for first prescriptions (aOR } 5.1,95 \% \mathrm{Cl} \text { I.0-3I.7) and during the first month of treatment } \\
\text { (aOR 4.I, } 95 \% \mathrm{Cl} 0.7-23.9 \text { ). Stratification analysis according to indication of use showed that current use of } \\
\alpha \text {-blockers was not associated with hip/femur fracture in men with a diagnosis of benign prostatic hyperplasia, but } \\
\text { was associated in men who used } \alpha \text {-blockers for cardiovascular disease (aOR } 2.8,95 \% \mathrm{Cl} 1.4-5.4) .{ }^{17} \mathrm{No} \text { increased } \\
\text { risk of fractures was associated with current use of modified-release doxazosin in hypertension, previous use, or } \\
\text { the start of a treatment episode ( } \leq 28 \text { days). }{ }^{18} \text { Caution with respect to first-dose effects related to the initiation of } \\
\text { a new episode of } \alpha \text {-blocker treatment is advised, and modified-release doxazosin is a superior } \alpha \text {-blocker versus } \\
\text { standard formulation for the sake of avoiding fall risk in elderly patients. }\end{array}$ \\
\hline Digoxin & $\begin{array}{l}\text { Digoxin therapy ( } 35 \% \text { versus } 22 \% \text { ) was more common in hospitalized elderly patients who had fallen than in control } \\
\text { patients. }{ }^{19}\end{array}$ \\
\hline Miscellaneous & $\begin{array}{l}\text { Use of diabetes medications was significantly associated with an increased risk of falling (aOR } 3.2,95 \% \mathrm{CI} I .3-7.9)^{20} \\
\text { The rate of fall-related injuries for patients receiving a doublet of neurotoxic chemotherapy }(9.15 \text { per I,000 person- } \\
\text { months) was significantly higher than for those receiving a single neurotoxic agent ( } 7.76 \text { per I,000 person-months) } \\
\text { or a nonneurotoxic agent ( } 5.19 \text { per I,000 person-months). }{ }^{7} \text { Nasal preparations (eg, treatment of asthma or allergic } \\
\text { rhinitis) (aOR I.49, } 95 \% \mathrm{Cl} \text { I.07-2.08) and antiglaucoma ophthalmic preparations (aOR I.5I, } 95 \% \mathrm{Cl} \text { I. I0-2.09) } \\
\text { were statistically significantly associated with an emergency department visit due to a recurrent fall. }{ }^{5}\end{array}$ \\
\hline
\end{tabular}

Abbreviations: CNS, central nervous system (CNS-acting agents include benzodiazepines, sedatives, hypnotics, antidepressants, antipsychotic drugs, and anti-Parkinson drugs); NSAIDs, nonsteroidal anti-inflammatory drugs; OR, odds ratio; aOR, adjusted OR; HR, hazard ratio; $\mathrm{Cl}$, confidence interval.

\section{Effects of drug PK/PD properties on fall risk}

\section{Elimination half-life}

Benzodiazepines are classified into three groups according to elimination half-life: 1) long-acting agents with half-life exceeding 24 hours (diazepam, delorazepam, flunitrazepam, and nitrazepam);2) intermediate and short-acting agents with half-life between 5 and 24 hours (lorazepam, bromazepam, oxazepam, and alprazolam); and 3) ultra-short-acting agents with very short half-life less than 5 hours (triazolam and brotizolam). ${ }^{21}$
Multivariate analysis of risk factors for falls in 7,908 hospitalized patients showed that benzodiazepines with very short and short half-life were positively associated with falls during hospital stays. ${ }^{22}$ The odds ratios (ORs) were 1.9 (95\% confidence interval [CI] 1.03-3.3, $P<0.05)$ and $1.8(95 \%$ CI $1.2-2.8, P<0.01)$, respectively. Interestingly, long half-life benzodiazepines did not exhibit a significant positive association with the risk of falls (OR 0.8, 95\% CI 0.4-1.8). This indicates that the use of benzodiazepines with short or very short half-life is an important and independent risk factor for falls, and 
careful evaluation should be given to their prescriptions to elderly inpatients.

Wang et al reviewed the health care utilization data of 1,222 hip fracture patients and 4,888 matched control patients aged 65 years and over, and found that use of benzodiazepines other than long-acting agents significantly increased the risk of hip fracture by $50 \% .{ }^{23}$ Very recently, de Vries et al conducted two prospective observational studies to investigate whether long-acting benzodiazepines were associated with a higher fall risk than short-acting agents. The primary endpoints were time to the first fall after inclusion and number of falls in the first year after inclusion. The use of short-acting benzodiazepines was associated with time to the first fall (hazard ratio [HR] 1.62 [95\% CI 1.03-2.56] and HR 1.64 [95\% CI 1.19-2.26], respectively) and number of falls (OR 1.28 [95\% CI 1.01-1.61] and OR 1.37 [95\% CI 1.10-1.70]). On the contrary, long-acting benzodiazepines were not significantly associated with time to first fall or number of falls. ${ }^{24}$ However, long-acting benzodiazepines (but not short-acting benzodiazepines) have been described as potentially inappropriate in Screening Tool of Older Persons' Prescriptions (STOPP) criteria. ${ }^{25}$ Therefore, there might be the need to regularly revise and update these criteria.

\section{Metabolic pathway}

Benzodiazepines are classified into two groups according to their drug-metabolism profile: 1) nonoxidative benzodiazepines that are primarily metabolized via uridine 5'-diphospho-glucuronosyltransferase-mediated glucuronidation (eg, lorazepam, oxazepam, and temazepam); and 2) oxidative benzodiazepines that are primarily metabolized by cytochrome P450 (CYP) isoenzymes (eg, alprazolam, chlordiazepoxide, clorazepate, diazepam, estazolam, flurazepam, halazepam, prazepam, quazepam, and triazolam). ${ }^{26}$

Sgadari et al found that among patients receiving oxidative benzodiazepines at high dosages, the risk of hip fracture was somewhat different depending on age: OR 0.87 (95\% CI 0.41-1.84) for patients aged 65-74 years, OR $1.15(95 \% \mathrm{CI}$ $0.75-1.56)$ for patients aged 75-84 years, and OR $1.55(95 \%$ CI 1.5-2.28) for those aged 85 years or older. A similar age dependency was observed in those taking oxidative benzodiazepines on an as-needed basis. In contrast, among people taking nonoxidative benzodiazepines, the relationship between age and risk of femur fracture exhibited a flat curve regardless of dosage. ${ }^{27}$ When the analysis was restricted to the only two comparable groups (ie, short half-life agents), the overall difference between the estimates of the effect for oxidative and nonoxidative benzodiazepines remained.
Therefore, oxidative benzodiazepines seem to be more harmful than nonoxidative ones, but only among the oldest patients receiving higher dosages. The underlying mechanism for this finding may be that the hepatic oxidative capacity could be exceeded in the oldest individuals and in those receiving high dosages, resulting in drug accumulation and an increase in the likelihood of adverse events.

\section{Genetic polymorphism}

Generally, in the field of pharmacokinetics/pharmacodynamics, there is a relationship between the drug dose (or the plasma concentration) and the pharmacologic effect of the drug, so it is supposed that there may be a relation between blood level of FRIDs and fall risk. It seems interesting to investigate whether a decreased metabolism or efflux of FRID due to polymorphisms of genes encoding for CYP enzymes or drug transporters is associated with an increased fall risk.

Blonk et al innovatively examined the association between CYP2D6*4, CYP3A5*3, and ABCB1 3435T polymorphisms and fall risk in elderly patients using FRID substrates of CYP2D6, CYP3A5, or P-glycoprotein (P-gp). Among the CYP2D6 subgroup (ie, 57 users of CYP2D6 substrates), the OR for risk of falling showed a statistically nonsignificant increase in four poor metabolizers (PMs) compared to 53 participants who were extensive metabolizers (EMs) or intermediate metabolizers (IMs) (OR 2.2, 95\% CI 0.2-25.0). A similar result was observed in the P-gp subgroup (3435TT versus $3435 \mathrm{CC}+3435 \mathrm{CT})$, with an OR of 2.1 (95\% CI 0.2-17.2). For users of CYP3A5 substrates, there appeared to be no difference in fall risk between PMs $(n=49)$ and EMs + IMs ( $\mathrm{n}=14)\left(\mathrm{OR} 0.9,95 \%\right.$ CI 0.2-3.3). ${ }^{28}$ The lack of statistical significance of the increased OR seen in the CYP2D6 and P-gp subgroup might be due to the fact that the cohorts were insufficiently powered for the subanalysis. It is worth conducting a prospective study in a larger population to confirm the possible association of genetic polymorphism with fall risk in the elderly.

\section{Risk rating of medications despite belonging to the same therapeutic class \\ Antihypertensives}

Gribbin et al estimated adjusted ORs for ever-exposure and current/previous exposure to the main classes of antihypertensives in older people with a recorded fall in primary care, and also examined the effect of the time interval from first prescription to first fall. They classified exposure to each class of drugs in terms of the elapsed time between the index event and final preceding prescription, as follows: 
ever prescribed, current (last prescription within 60 days of index event), recent (60-120 days), previous (more than 120 days), or never prescribed. Results of that case-control study showed strong positive associations at 0 days for all classes of medication ( $\beta$-blockers, angiotensin-converting enzyme inhibitors, calcium antagonists, thiazides). Surprisingly, only thiazides showed significant ORs of falling during elapsed times of 1-7, 8-14, and 15-21 days following the first prescription. The adjusted ORs of first fall for thiazides were as follows: days $1-7$ (OR 5.41, 95\% CI 1.62-18.14), days 8-14 (OR 5.02, 95\% CI 1.63-15.51), and days 15-21 (OR 4.28, 95\% CI 1.19-15.42). ${ }^{16}$

A study showed that the current prescribing of angiotensin system-blocking medications, including angiotensin-converting enzyme inhibitors and angiotensinreceptor blockers, had a protective effect against fall risk in older people..$^{29}$ This prospective cohort study in 520 community-dwelling older people indicated that people taking angiotensin system-blocking medications were less likely to fall (OR $0.68,95 \%$ CI $0.48-0.97 ; P<0.05$ ) after adjusting for potential confounders. The mechanisms for this apparent protective effect are unclear and worthy of further investigation.

\section{Analgesics}

A systematic review in 2009 suggested that an increased risk for accidental falls is probable when elderly individuals are exposed to nonsteroidal anti-inflammatory drugs (NSAIDs). ${ }^{30}$ With respect to the fall risk of other analgesics, meta-analysis has not been available. Analgesic-prescribing habits may differ among physicians. Rolita et al conducted a nested case-control study in an elderly population suffering from osteoarthritis to examine the incidence of falls and fractures in relation to analgesics prescribed: narcotics (opioids), cyclooxygenase-2 inhibitors, and NSAIDs. Narcotic analgesic prescriptions were associated with a significantly greater risk of falls and fractures. The likelihood of experiencing a fall/fracture was higher in patients receiving narcotic analgesics than those receiving a cyclooxygenase-2 inhibitor (OR 3.3, 95\% CI 2.5-4.3; $P<0.001$ ) or NSAID (OR $4.1,95 \%$ CI $3.7-4.5 ; P<0.001) .{ }^{31}$ For older adult patients with chronic pain at higher risk for NSAID-related adverse effects, such as those who have gastrointestinal or cardiovascular disease, diabetes mellitus, or who are taking low-dose aspirin, narcotics are recommended instead. ${ }^{32}$ It is worth reevaluating such recommendations, considering the increased fall risk caused by the use of narcotic analgesics.

Walker et al observed that NSAIDs were administered more frequently to hospitalized elderly patients who fell (48\%) than to matched control patients who did not fall (18\%) $(P=0.002)$, and NSAID use was associated with a tenfold increase in the likelihood of falling in hospitalized elderly patients (OR 10.02, 95\% CI 2.6-38.58; $P=0.001$ ), whereas opioid analgesics were given more frequently to control patients $(68 \%)$ than to patients who fell $(31 \%)(P<0.001) .{ }^{14}$ The authors acknowledged several confounding factors in the study, ie, uncontrolled differences in nursing care or fall-prevention efforts and uncontrolled health status, which may have led to overestimation of the risk of falling with NSAIDs. The fact that opioid analgesics are not associated with an increased fall risk may be attributed to the fact that the need for fall prevention might have been anticipated in patients receiving opioids, because the risk of falling with opioids is well known.

\section{Effects of characteristics of medication use on fall risk} Number of medications and drug-drug interaction

In geriatric outpatients, polypharmacy rather than number of comorbidities was associated with fall risk, and the association of number of medications with fall risk was stepwise. Taking five or more drugs was significantly associated with an increased risk of falls in geriatric outpatients (OR 4.5, 95\% CI 1.7-12.2) after adjusting for potential confounders. ${ }^{33,34}$ Outpatients would experience a $14 \%$ increase in fall risk with the addition of each medication beyond a four-medication regimen (OR 1.14, 95\% CI 1.02-1.27; $P=0.027) .{ }^{35}$ Mizukami et al examined the prevalence of falls among communitydwelling elderly Japanese individuals, and found that taking at least four daily prescription medications $(P<0.05)$ was significantly associated with falls. ${ }^{36}$

Multivariate logistic regression analysis revealed that the overall OR of falling among patients using ten or more drugs was 8.42 (95\% CI 4.73-15.0) compared with patients who used $0-1$ drug per day. ${ }^{37}$ Another study also showed particular risk for falling (OR 6.1) in patients taking 10+ drugs. ${ }^{38}$ Patients who are taking many different types of medications are high-risk groups, where increased monitoring or tailored drug regimens is necessary.

In a prospective cohort study of 204 patients (mean age $80.5 \pm 8.3$ years) admitted to hospital after a fall, frail participants $(n=103)$ had a significantly higher number of FRIDs on admission compared with robust patients $(\mathrm{n}=101)$ (frail 3.4 \pm 2.2 versus robust $1.6 \pm 1.5, P<0.0001$ ), total number of medications $(9.8 \pm 4.3$ versus $4.4 \pm 3.3, P<0.0001)$, and DDI exposure ( $35 \%$ versus $5 \%, P=0.001)$. The number of FRIDs and to a lesser extent total medications at discharge 
were significantly associated with recurrent falls (OR 1.7 and OR 1.2, respectively), but DDIs were not. ${ }^{39}$

French et al analyzed 133,872 outpatient benzodiazepine prescriptions and $>1.5$ million nonbenzodiazepine prescriptions. Micromedex ${ }^{\circledR}$ software (Truven Health Analytics, Rocky Hill, CT, USA) was used to identify combinations of benzodiazepines and other drugs that are likely to result in "major" interactions; $71.2 \%$ (790 of 1,110$)$ of the unique patients who experienced an injury had used benzodiazepine in combination with another drug with a major severity rating defined by Micromedex. Interestingly, the occurrence rate of fall incidents was only 4.3\% (320 of 7,522) among patients who were taking benzodiazepines without concomitant use of precipitant drugs, whereas concomitant use of benzodiazepines and precipitant drugs increased the odds of an injury more than twofold. ${ }^{40}$

Some chemotherapeutic agents, such as platinum compounds, taxanes, and vinca alkaloids, may be neurotoxic and lead to peripheral neuropathy, which can cause and exacerbate balance and gait problems and are associated with a two- to threefold increased risk of falling in community-dwelling elderly patients. A retrospective cohort analysis using the records of 65,311 patients with breast, colon, lung, or prostate cancer treated with chemotherapy revealed that the rate of fall-related injuries for patients receiving a doublet of neurotoxic chemotherapy (9.15 per 1,000 person-months) was significantly higher than for those receiving a single neurotoxic agent (7.76 per 1,000 person-months) or a nonneurotoxic agent (5.19 per 1,000 person-months). ${ }^{7}$

\section{Dose strength}

The effect of dose strength on fall risk was mainly observed with CNS agents. In a case-control study, multivariate logistic regression showed benzodiazepine doses $\geq 1 \mathrm{mg}$ /day in diazepam equivalents (OR 2.14, 95\% CI 1.04-4.39; $P<0.05$ ) was significantly associated with falls among hospitalized older people, whereas the OR for falls at a diazepam equivalent dose of less than $1 \mathrm{mg}$ /day was $0.96 .{ }^{42}$ Herings et al reported a strong dose effect of benzodiazepines on the occurrence of accidental falls leading to hospitalization for femur fractures $(P<0.0001){ }^{42}$

Sterke et al evaluated the dose-response relationship between psychotropic drugs and falls in nursing home residents with dementia. Significant dose-response relationships were observed with the use of antipsychotics (HR 2.78), anxiolytics (HR 1.60), hypnotics and sedatives (HR 2.58), and antidepressants (HR 2.84). Fall risk increased significantly with $28 \%$ at 0.25 of the defined daily dose (DDD) of an antipsychotic or antidepressant, with $8 \%$ at 0.2 of the DDD of an anxiolytic, and with $56 \%$ at 0.5 of the DDD of a hypnotic or sedative; it increased further with dose increases and with combinations of psychotropics. ${ }^{43}$

Hanlon et al reported that there was a remarkable doseresponse relationship between total CNS medication dose and recurrent (two or more) falls in older adults. A nearly threefold-increased risk of recurrent falls (adjusted OR [aOR] $2.89,95 \%$ CI 1.96-4.25) was observed with patients taking higher doses ( $>3$ summated standard daily dose [SDD]), compared with risk of recurrent falls in those taking moderate doses (1-3 SDD) (aOR 1.80, 95\% CI 1.31-2.47) and lowest doses (<1.0 SDD) (aOR 1.42, 95\% CI 0.95-2.15). ${ }^{44}$

A population-based cohort study by Coupland et al showed significant association of falls in elderly patients with depression $(P<0.01)$ with doses of tricyclic antidepressants (TCAs) and selective serotonin-reuptake inhibitors (SSRIs). ${ }^{45}$ Sano et al observed an increased risk of falls in hospitalized psychiatric patients taking doses of chlorpromazine equivalents $\geq 600 \mathrm{mg}$ (OR $3.75,95 \%$ CI $2.41-5.83 ; P<0.05$ ) or doses of diazepam equivalents $\geq 15 \mathrm{mg}$ (OR 2.08, 95\% CI 1.45-2.98; $P<0.05) .{ }^{46}$ Sterke et al found a significant dose-response relationship between injurious falls and the use of SSRIs. The risk of an injurious fall increased significantly with $31 \%$ at 0.25 of the DDD of an SSRI, 73\% at $0.50 \mathrm{DDD}$, and $198 \%$ at $1.00 \mathrm{DDD}$ (HR 2.98, 95\% CI 1.94-4.57). Even at low doses, SSRIs were associated with increased risk of an injurious fall in nursing home residents with dementia. Higher doses increased the risk further, with a threefold risk at 1.00 DDD. ${ }^{47}$

There was little literature on the dose-response relationship between fall risk and the use of non-CNS medications. Berry et al reported that increasing the dose of loop diuretic drugs could result in an elevated risk of falls in nursing home residents. ${ }^{15}$ Bauer et al analyzed the dose-response relationship between antiarrhythmics and injuries, including drug consumption measured in number of DDDs, and found that the incidence of injuries strongly increased with doses of antiarrhythmics. In the case of the zero-inflated negative binomial regression model, the results showed that the number of injuries increased by 0.0020 with an increase of one prescribed DDD. ${ }^{48}$

\section{Duration of medication use and time since stopping}

Duration of medication use and time since stopping are important factors determining the magnitude of fall risk. A nested case-control study by Modén et al investigated the associations between use of psychotropic drugs and falling 
accidents in a population aged 65 years and older. Interestingly, they found that the period following the first prescription to the time of falling was closely related with fall risk. Using psychotropic drugs within 3 months before the fall was associated with more than doubled odds for a fall in all participants. This effect seemed to be strongest immediately after initiating therapy. The use of psychotropic drugs during the week before the accident occurred was associated with even higher odds for a fall among both men (OR 5.61, 95\% CI 2.54-12.41) and women (OR 3.40, 95\% CI 2.24-5.17). ${ }^{49}$

A retrospective cohort study by Mehta et al showed that duration of therapy with any antipsychotic medication for $>90$ days was significantly associated with increased risk of falls/fractures compared with $<30$ days of treatment (HR $1.81,95 \%$ CI $1.35-2.43) .{ }^{50}$ Coupland et al analyzed the association of antidepressant use with risk of adverse outcomes in older people by a population-based cohort study. Adjusted HRs for falls were closely associated with duration of antidepressant use and time since stopping. The rate of falls was highest in the first 28 days after starting an antidepressant, and also in the first 28 days after stopping. ${ }^{45}$ Adjusted HRs for falls in patients receiving TCAs versus patients not currently on antidepressants were as follows: 1-28 days (HR 1.48), 29-84 days (HR 1.16), 85+ days (HR 1.10), stopped 1-28 days (HR 3.62), stopped 29-84 days (HR 1.47), and stopped 85-182 days (HR 1.08). Adjusted HRs for falls in patients receiving SSRIs versus patients not currently on antidepressants were as follows: 1-28 days (HR 2.23), 29-84 days (HR 2.20), 85+ days (HR 1.38), stopped 1-28 days (HR 5.03), stopped 29-84 days (HR 1.47), and stopped 85-182 days (HR 1.09).

\section{Medication change}

Sorock et al investigated the role of changes in six medication categories on the risk of falling in nursing home residents (mean age 80.5 years). The risk of falls was significantly elevated within 3 days of a CNS medication change (ie, any new medication, dosage change, as needed, or discontinued medication). The fall risk statistically significantly increased 3.4-fold (95\% CI 1.2-9.5) within 1-3 days following a change in any CNS medication. No changes in other medication categories (eg, gastrointestinal, hypoglycemics, antibiotics, cardiovascular disease agents, and analgesics) had a significant effect on fall risk. ${ }^{51}$

A retrospective case-cohort study of 39,813 patients aged $>65$ years concluded that recent changes in cardiovascular or psychotropic medications were associated with an increased risk of subsequent admission for falls or fractures (OR 1.54, 95\% CI 1.17-2.03, and OR 1.68, 95\% CI 1.28-2.22, respectively). The strongest associations were observed for changes in SSRI antidepressant (OR 1.99, 95\% CI 1.29-3.08), non-SSRI/TCA (OR 4.39, 95\% CI 2.21-8.71), and combination psychotropic medication (OR 3.05, 95\% CI 1.66-5.63). ${ }^{52}$

A prospective cohort study of nursing home residents showed that the proportion of residents with two or more falls during follow-up was greater among residents with a psychotropic drug change compared to psychotropic users without change $(41.0 \%$ versus $22.5 \%, P<0.001) .{ }^{53}$ Medication change of a psychotropic drug within 24 hours (aOR 10.3 , 95\% CI 1.37-76.8) was an important variable significantly associated with an increased risk of falling in nursing home residents. ${ }^{54}$

Berry et al observed that changes in specifically nonSSRI antidepressant prescriptions increased the risk of falls within 2 days (OR 4.7, 95\% CI 1.3-16.2), with a subsequent decreased risk of falls over the next 5 days (OR 1.9, 95\% CI 0.9-4.0). ${ }^{55}$ Increased staff awareness might help to reduce falls during this time, and surveillance should be strengthened particularly during the first 48 hours.

Nursing home residents are at an increased risk of falls in the day following a new prescription or increased dose of a loop diuretic drug (OR 2.46, 95\% CI 1.02-5.92). However, there was no evidence of an increased risk of falls when the hazard period was extended beyond 1 day following a loop diuretic drug change (OR for days $2-7,1.13-1.57) .{ }^{15}$

Of the 543,572 new users of antihypertensive drugs among community-dwelling elderly, 8,893 experienced an injurious fall that required hospital care. New users had a $69 \%$ increased risk of having an injurious fall during the first 45 days following antihypertensive treatment (95\% CI, $1.57-1.81) .^{56}$

Medication reconciliation is vital in preventing adverse events in care transition (eg, on admission, at discharge), where medication changes usually occur among patients with polypharmacy; therefore, it is worth conducting further study to investigate whether medication reconciliation would decrease the risk of falling in the elderly.

\section{Prescribing appropriateness}

Berdot et al evaluated the associations between the use of potentially inappropriate medications (PIMs) and the risk of falls by a prospective study in a large community-dwelling elderly cohort. Overall, inappropriate medication users had an increased risk of falling, which was mainly due to the use of long-acting benzodiazepines (aOR 1.4, 95\% CI 1.1-1.8) 
in both occasional and regular users, other inappropriate psychotropics (aOR 1.7, 95\% CI 1.7-2.7) in regular users, or medication with anticholinergic properties (aOR 1.6, 95\% CI 1.2-2.1) in regular users. ${ }^{57}$

The multivariate analyses indicated a relation between PIM-related acute admissions in frail older persons (median age 84 years) and a history of previous falls $(P<0.001)$. PIM use, with $48 \%$ prevalence, mainly involved overuse and/or misuse of benzodiazepines, aspirin, and opiates according to the STOPP criteria. ${ }^{58,59}$ Pharmacological optimization approaches should be taken to address the inappropriate prescribing.

A study by Agashivala and Wu showed a 20.5\% increased risk among elderly US nursing home residents taking potentially inappropriate psychoactive medications compared with those taking other psychoactive medications, and a $60.3 \%$ increased risk compared with those taking nonpsychoactive medications. ${ }^{59}$ This indicates that use of potentially inappropriate psychoactive medications poses the potential threat of falls among elderly nursing home residents.

A prospective, observational cohort study of 4,260 community-dwelling older men (mean age $77 \pm 3.6$ years) showed that the number of PIMs (OR 1.23, 95\% CI 1.04-1.45; $P=0.018$ ) was associated with self-reported history of falls in the previous 12 months. PIM use included self-reported use of a medication commonly contraindicated in the elderly, a medication which is commonly toxic among the elderly, or medications suggesting prescribing cascade (cholinesterase inhibitors with systemic anticholinergics, anti-Parkinson's medications with dopamine antagonists, diuretics with NSAIDs, thiazides with antigout therapy, $\beta$-blockers with selfreported history of asthma). ${ }^{60}$ Modifying polypharmacy and optimizing effective medications may be effective approaches for reducing the risk of adverse health outcomes.

McMahon et al found that a significant prevalence of PIM use was observed in older fallers presenting to the emergency department. No substantial improvements in PIM use occurred in the 12 months after the fall, suggesting the need for focused intervention studies to be undertaken in this area. ${ }^{61}$

\section{Medication adherence}

Poor medication adherence may occur in as many as $40 \%-50 \%$ of community-dwelling elders. Important factors that influence adherence include forgetfulness, lack of interest or knowledge, medication expense, complicity in medication regimens, and limited access to health care providers.

Berry et al creatively investigated whether poor medication adherence would increase the rate of falls among community-dwelling elders. A total of 246 men and 408 women (mean age 78 years) were followed for the occurrence of falls. A participant was characterized as having low medication adherence if he or she answered yes to any of the following questions: Do you ever forget to take your medications? Are you careless at times about taking your medications? When you feel better, do you sometimes stop taking your medications? Sometimes, if you feel worse when taking your medication, do you stop taking it? High adherence was defined as a "no" answer to every question. Subjects with low medication adherence were more likely to experience two or more falls compared with subjects with high adherence $(44 \%$ versus $28 \%, P<0.001)$. The rate of falls in the low-adherence group was 1.1 falls/person-year $(95 \%$ CI 1.0-1.3) compared with 0.7 falls/person-year (95\% CI 0.6-0.8) in the high-adherence group. Low medication adherence was associated with an increased risk of falls compared with high medication adherence (OR 1.5, 95\% CI 1.2-1.9; $P<0.001)$ after adjusting for age, sex, race/ethnicity, education, alcohol use, cognitive measures, functional status, depression, and number of medications. ${ }^{62}$ The underlying mechanism for this new finding is unclear. Low medication adherence is supposed to accompany higher odds of medication changes, which are associated with an increased risk of falling for some drug classes. The association of falls with poor adherence to use of specific drug classes or with reasons for poor medication adherence has not been addressed, and should be further examined. Clinicians should screen for poor medication adherence, as it is common and predicts falls, despite the fact that it remains unclear whether improving medication adherence could decrease the rate of falls.

Ruiz et al found that noncompliance with medications was directly correlated with fall risk $(P<0.001)$ in human immunodeficiency virus-positive patients. Possible explanations for this finding may be as follows: 1) drug and substance abuse that may put these patients at a higher risk of falls; 2) DDIs or adverse drug reactions that prevented patients from continuing taking their medications; and 3) possible immune system deterioration that in turn may predispose patients to develop further complications, including falls. ${ }^{63}$

\section{Effects of pharmacological interventions on fall risk Withdrawal of FRIDs}

Evidence showed that withdrawal of FRIDs in geriatric outpatients could generate significant cost savings. ${ }^{64}$ In a geriatric outpatient population in the Netherlands, patients with a history of falls were assessed for fall risk $(n=139)$. FRIDs 
were withdrawn when appropriate $(n=75)$. All participants had a 2-month follow-up for fall incidents. The total number of fall incidents during follow-up was significantly lower in these 75 patients than in those who continued treatment (mean number of falls, 0.3 versus $3.6 ; P=0.025)$. The adjusted HR of a fall during follow-up was 0.48 (95\% CI $0.23-0.99)$ for overall drug withdrawal, 0.35 (95\% CI 0.15-0.82) for cardiovascular drug withdrawal, and 0.56 (95\% CI $0.23-1.38)$ for psychotropic drug withdrawal. The withdrawal of cardiovascular medication achieved the most marked results. ${ }^{65}$ Net cost savings were $€ 1,691$ per patient in the cohort. Extrapolating this finding to the national scale in the Netherlands, the authors anticipated a reduction of $€ 60$ million in health care expenditures (ie, $15 \%$ of fall-related health costs). ${ }^{64}$

Withdrawing hypnosedative medications is challenging, due to their propensity for causing dependence and rebound insomnia. Patients who were assessed by a geriatrician were more likely to be advised to alter their benzodiazepine use than those assessed by a rehabilitation specialist $(P=0.002)$. Patients were more likely to be compliant if they were given a recommendation to cease the use of benzodiazepines or Z-drugs (eg, zaleplon, zolpidem, or zopiclone) compared with advice to reduce the dose or to gradually reduce the dose with the aim of cessation $(P=0.019) .{ }^{66}$

\section{Pharmacist-conducted clinical medication review}

Medication reviews have been identified as a key component of fall prevention. Kelly et al offered a consistent framework for conducting medication reviews and reducing fall risk. ${ }^{67}$ Pharmacists should accurately identify all current medications given to the individual patient, and consider the appropriateness of each medication use, potential or actual adverse drug reactions, and compliance issues. When a medication has been identified as nonessential or not indicated, discontinuation should be considered. If the medication is classified as important but may also increase the risk of falls, it may be possible to seek a safer alternative or try to reduce dosage.

A randomized controlled trial proved the beneficial impact of pharmacist-conducted clinical medication review. A total of 661 elderly care-home residents on one or more medications were recruited. In the intervention group, a pharmacist reviewed clinical medications and clinical records, and provided recommendations to a general practitioner for approval and implementation. Control patients received usual general practitioner care. During the intervention period, $75.6 \%$ of pharmacist recommendations were accepted by the general practitioner.
After 6 months' intervention, the mean number of medication changes per patient was 3.1 for intervention and 2.4 for the control group $(P<0.0001)$, and there were 0.8 and 1.3 falls per patient, respectively $(P<0.0001){ }^{68}$

Ferreri et al published a methodology of a randomized controlled trial to prevent falls through enhanced pharmaceutical care. ${ }^{69}$ Individuals in the intervention group were invited to attend a face-to-face medication consultation provided by a community pharmacy resident (ie, identification of drugtherapy problems and therapeutic recommendations), whereas those in the control group did not receive medication consultation. All participants were followed up for 24 months. The primary study endpoints included time to first fall and proportion of participants who experienced one or more falls during the first year of follow-up. Although there was no significant reduction in the rate of recurrent falls, injurious falls, or overall use of high-risk medications, individuals in the intervention group were more likely to discontinue use of a high-risk medication or have the dosage reduced during the 1-year follow-up period compared with those in the control group. ${ }^{70}$

Haumschild et al described the effects of a 1-year fall-focused pharmaceutical intervention program on the clinical and economic outcomes of elderly patients in a rehabilitation center. Two hundred patients were randomly selected from the preintervention phase and postintervention phase. The pharmaceutical intervention included a complete review of all medications by a consultant pharmacist. Any medications identified as causing a particular adverse effect (eg, dizziness) or clinical condition (eg, falls and fractures) were listed in table format for review by the pharmacist, nurse, and physician. Written recommendations for dosage reduction and frequency adjustment were made. Precautions for drug administration were given to nursing personnel and attached to the patients' medication-administration records within 24 hours of admission to the rehabilitation center. The consultant pharmacist and nurse immediately implemented the pharmaceutical interventions in the patient's plan of care after collaborating with the physician to obtain medical orders relevant to the interventions. The number of falls was reduced in the postintervention group by $47 \%$, resulting in future savings of US\$7.74 per patient per day. The use of the following drug classes decreased in the postintervention period: cardiovascular agents, $10.7 \%$; analgesics, $6.3 \%$; psychoactive drugs, $18.2 \%$; and sedatives and hypnotics, $13.9 \%{ }^{71}$

\section{Computerized drug alerts}

Computerized drug alerts are expected to reduce fall-related injuries in older patients. However, physicians may ignore 
most alerts, because they believe the benefit of the drugs outweighs the risk. Tamblyn et al conducted a cluster-randomized trial to determine whether computerized prescribing decision support with patient-specific risk assessments would improve physician response to psychotropic drug alerts and decrease injury risk in the elderly. Eighty-one family physicians and 5,628 of their patients aged 65 years and older were recruited. Intervention physicians received an alert to patient-specific risk of injury when a patient was prescribed a psychotropic medication that increased the risk of injury. Risk thermometers presented changes in absolute and relative risk with each change in drug treatment. Control physicians received commercial drug alerts, including potential contraindications, therapy duplication, dosing error, cumulative toxicity, and drug-disease, drug-drug, and drug-allergy interactions. The intervention reduced the risk of injury by 1.7 injuries per $1,000$ patients (95\% CI $0.2 / 1,000-3.2 / 1,000, P=0.02)$. The effect of the intervention was greater for patients with higher baseline risks of injury $(P<0.03) .{ }^{72}$ A study by Tzeng et al determined the correlations between hospital-acquired injurious fall rates in US acute care hospitals and these institutions' implementation levels of computerized systems. The results showed that computerized decision-support systems for DDI alerts, drug allergy alerts, and drug-laboratory interaction alerts were effective to inform practice for better interventions to reduce fall risk. ${ }^{73}$

There are limitations of this review. We did not summarize medications that would reduce fall risk in elderly patients. For example, vitamin D could decrease falls and hip fractures in patients with vascular parkinsonism by increasing muscle strength. ${ }^{74}$ We did not present our paper in the form of a Cochrane-style systematic review or meta-analysis, which can resolve limitations due to sample size. However, a new horizon might be opened by this review despite some limitations. Further studies should be conducted in this area, such as investigating whether medication reconciliation and improving medication adherence could decrease the rate of falls. Sample stratification and better controlling for potential confounders are encouraged in relevant studies of medication-related fall risk.

\section{Conclusion}

Clinicians who take care of elderly people should be aware of which drugs are FRIDs, and how PK/PD properties of FRIDs, medication use characteristics, and pharmacological interventions exert influences on fall risk in elderly patients. Clear practical recommendations to prevent falls in elderly based on the literature review are:
- make a list of FRIDs for clinicians

- establish a computerized alert system for when to prescribe FRIDs through an electronic medical record system

- seek an alternative with lower fall risk

- withdraw FRIDs if clinically indicated, and take pertinent caution when the use of FRIDs cannot be avoided

- pay attention to prescribing appropriateness, simplify the medication regimen, and avoid adverse DDIs

- strengthen pharmacist-conducted clinical medication review

- ensure the label of each FRID dispensed contains corresponding warning sign ${ }^{75}$

- be careful when medication change occurs

- enhance medication adherence

- mandate for periodic reassessment of the potential risk associated with the patient's medication regimen.

\section{Acknowledgments}

This work was supported by the Zhejiang Provincial Bureau of Health (grant 2012KYA090 and 2013KYB138), the National Natural Science Foundation of China (grant 81373488), and the National Major Projects of China (grants 2012ZX09506001-004).

\section{Disclosure}

The authors report no conflicts of interest in this work.

\section{References}

1. World Health Organization. WHO Global Report on Falls Prevention in Older Age. Geneva: WHO; 2007. Available from: http://www.who.int/ageing/publications/Falls_prevention7March.pdf. Accessed May 20, 2014.

2. Currie L. Fall and injury prevention. In: Hughes RG, editor. Patient Safety and Quality: An Evidence-Based Handbook for Nurses. Rockville (MD): Agency for Healthcare Research and Quality; 2008:1-195-1-250. Available from: http://www.ncbi.nlm.nih.gov/books/NBK2653. Accessed May 20, 2014.

3. Joint Commission International. Joint Commission International Accreditation Standards for Hospitals. 2014. Available from: http://www. onlinedic.net/docs/JCI_5th_Edition.pdf. Accessed June 2, 2014.

4. Huang AR, Mallet L, Rochefort CM, Eguale T, Buckeridge DL, Tamblyn R. Medication-related falls in the elderly: causative factors and preventive strategies. Drugs Aging. 2012;29:359-376.

5. Askari M, Eslami S, Scheffer AC, et al. Different risk-increasing drugs in recurrent versus single fallers: are recurrent fallers a distinct population? Drugs Aging. 2013;30:845-851.

6. Epstein NU, Guo R, Farlow MR, Singh JP, Fisher M. Medication for Alzheimer's disease and associated fall hazard: a retrospective cohort study from the Alzheimer's disease neuroimaging initiative. Drugs Aging. 2014;31:125-129.

7. Ward PR, Wong MD, Moore R, Naeim A. Fall-related injuries in elderly cancer patients treated with neurotoxic chemotherapy: a retrospective cohort study. J Geriatr Oncol. 2014;5:57-64.

8. Costa-Dias MJ, Oliveira AS, Martins T, et al. Medication fall risk in old hospitalized patients: a retrospective study. Nurse Educ Today. 2014;34:171-176. 
9. Lamis RL, Kramer JS, Hale LS, Zackula RE, Berg GM. Fall risk associated with inpatient medications. Am J Health Syst Pharm. 2012;69:1888-1894.

10. Shuto H, Imakyure O, Matsumoto J, et al. Medication use as a risk factor for inpatient falls in an acute care hospital: a case-crossover study. Br J Clin Pharmacol. 2010;69:535-542.

11. Rhalimi M, Helou R, Jaecker P. Medication use and increased risk of falls in hospitalized elderly patients: a retrospective, case-control study. Drugs Aging. 2009;26:847-852.

12. Tanaka M, Suemaru K, Ikegawa Y, Tabuchi N, Araki H. Relationship between the risk of falling and drugs in an academic hospital. Yakugaku Zasshi. 2008;128:1355-1361.

13. Mamun K, Lim JK. Association between falls and high-risk medication use in hospitalized Asian elderly patients. Geriatr Gerontol Int. 2009;9:276-281.

14. Walker PC, Alrawi A, Mitchell JF, Regal RE, Khanderia U. Medication use as a risk factor for falls among hospitalized elderly patients. Am J Health Syst Pharm. 2005;62:2495-2499.

15. Berry SD, Mittleman MA, Zhang Y, et al. New loop diuretic prescriptions may be an acute risk factor for falls in the nursing home Pharmacoepidemiol Drug Saf. 2012;21:560-563.

16. Gribbin J, Hubbard R, Gladman JR, Smith C, Lewis S. Risk of falls associated with antihypertensive medication: population-based casecontrol study. Age Ageing. 2010;39:592-597.

17. Souverein PC, Van Staa TP, Egberts AC, De la Rosette JJ, Cooper C, Leufkens HG. Use of $\alpha$-blockers and the risk of hip/femur fractures. J Intern Med. 2003;254:548-554.

18. Hall GC, McMahon AD. Comparative study of modified release alpha-blocker exposure in elderly patients with fractures. Pharmacoepidemiol Drug Saf. 2007;16:901-907.

19. Gales BJ, Menard SM. Relationship between the administration of selected medications and falls in hospitalized elderly patients. Ann Pharmacother. 1995;29:354-358.

20. Krauss MJ, Evanoff B, Hitcho E, et al. A case-control study of patient, medication, and care-related risk factors for inpatient falls. J Gen Intern Med. 2005;20:116-122.

21. Greenblatt DJ, Shader RI, Divoll M, Harmatz JS. Benzodiazepines: a summary of pharmacokinetic properties. Br J Clin Pharmacol 1981;11 Suppl 1:11S-16S

22. Passaro A, Volpato S, Romagnoni F, Manzoli N, Zuliani G, Fellin R. Benzodiazepines with different half-life and falling in a hospitalized population: the GIFA study. Gruppo Italiano di Farmacovigilanza nell'Anziano. J Clin Epidemiol. 2000;53:1222-1229.

23. Wang PS, Bohn RL, Glynn RJ, Mogun H, Avorn J. Hazardous benzodiazepine regimens in the elderly: effects of half-life, dosage, and duration on risk of hip fracture. Am J Psychiatry. 2001;158: 892-898.

24. de Vries OJ, Peeters G, Elders P, et al. The elimination half-life of benzodiazepines and fall risk: two prospective observational studies Age Ageing. 2013;42:764-770.

25. Gallagher P, Ryan C, Byrne S, Kennedy J, O'Mahony D. STOPP (Screening Tool of Older Persons' Prescriptions) and START (Screening Tool to Alert doctors to Right Treatment). Consensus validation. Int J Clin Pharm Ther. 2008;46:72-83.

26. de Leon J. Glucuronidation enzymes, genes and psychiatry. Int $J$ Neuropsychopharmacol. 2003;6:57-72.

27. Sgadari A, Lapane KL, Mor V, Landi F, Bernabei R, Gambassi G. Oxidative and nonoxidative benzodiazepines and the risk of femur fracture. The Systematic Assessment of Geriatric Drug Use Via Epidemiology Study Group. J Clin Psychopharmacol. 2000;20: 234-239.

28. Blonk MI, van der Velde N, van den Bemt PM, van Schaik RH, van der Cammen TJ. CYP2D6*4, CYP3A5*3 and ABCB1 3435T polymorphisms and drug-related falls in elderly people. Pharm World Sci. 2010;32:26-29.

29. Wong AK, Lord SR, Sturnieks DL, Delbaere K, Trollor JN, Close JC. Angiotensin system-blocking medications are associated with fewer falls over 12 months in community-dwelling older people. J Am Geriatr Soc. 2013;61:776-781.
30. Hegeman J, van den Bemt BJ, Duysens J, van Limbeek J. NSAIDs and the risk of accidental falls in the elderly: a systematic review. Drug Saf. 2009:32:489-498.

31. Rolita L, Spegman A, Tang X, Cronstein BN. Greater number of narcotic analgesic prescriptions for osteoarthritis is associated with falls and fractures in elderly adults. J Am Geriatr Soc. 2013;61: $335-340$.

32. de Leon-Casasola OA. Opioids for chronic pain: new evidence, new strategies, safe prescribing. Am J Med. 2013;126:S3-S11.

33. Kojima T, Akishita M, Nakamura T, et al. Association of polypharmacy with fall risk among geriatric outpatients. Geriatr Gerontol Int. 2011;11:438-444

34. Kojima T, Akishita M, Nakamura T, et al. Polypharmacy as a risk for fall occurrence in geriatric outpatients. Geriatr Gerontol Int. 2012; $12: 425-430$

35. Freeland KN, Thompson AN, Zhao Y, Leal JE, Mauldin PD, Moran WP. Medication use and associated risk of falling in a geriatric outpatient population. Ann Pharmacother. 2012;46:1188-1192.

36. Mizukami S, Arima K, Abe Y, et al. Falls are associated with stroke, arthritis and multiple medications among communitydwelling elderly persons in Japan. Tohoku J Exp Med. 2013;231: 299-303.

37. Lai SW, Liao KF, Liao CC, Muo CH, Liu CS, Sung FC. Polypharmacy correlates with increased risk for hip fracture in the elderly: a populationbased study. Medicine (Baltimore). 2010;89:295-299.

38. Neutel CI, Perry S, Maxwell C. Medication use and risk of falls. Pharmacoepidemiol Drug Saf. 2002;11:97-104.

39. Bennett A, Gnjidic D, Gillett M, et al. Prevalence and impact of fall-riskincreasing drugs, polypharmacy, and drug-drug interactions in robust versus frail hospitalised falls patients: a prospective cohort study. Drugs Aging. 2014;31:225-232.

40. French DD, Chirikos TN, Spehar A, Campbell R, Means H, Bulat T. Effect of concomitant use of benzodiazepines and other drugs on the risk of injury in a veterans population. Drug Saf. 2005;28: 1141-1150.

41. Chang CM, Chen MJ, Tsai CY, et al. Medical conditions and medications as risk factors of falls in the inpatient older people: a case-control study. Int J Geriatr Psychiatry. 2011;26:602-607.

42. Herings RM, Stricker BH, de Boer A, Bakker A, Sturmans F. Benzodiazepines and the risk of falling leading to femur fractures. Dosage more important than elimination half-life. Arch Intern Med. 1995;155:1801-1807.

43. Sterke CS, van Beeck EF, van der Velde N, et al. New insights: doseresponse relationship between psychotropic drugs and falls: a study in nursing home residents with dementia. J Clin Pharmacol. 2012;52: 947-955.

44. Hanlon JT, Boudreau RM, Roumani YF, et al. Number and dosage of central nervous system medications on recurrent falls in community elders: the Health, Aging and Body Composition study. J Gerontol A Biol Sci Med Sci. 2009;64:492-498

45. Coupland C, Dhiman P, Morriss R, Arthur A, Barton G, Hippisley-Cox J. Antidepressant use and risk of adverse outcomes in older people: population based cohort study. BMJ. 2011;343:d4551.

46. Sano T, Harada M, Sugawara T, et al. Use of psychotropics and the risk of falls in hospitalized psychiatric patients. Yakugaku Zasshi. 2013;133:897-903.

47. Sterke CS, Ziere G, van Beeck EF, Looman CW, van der Cammen TJ. Dose-response relationship between selective serotonin re-uptake inhibitors and injurious falls: a study in nursing home residents with dementia. Br J Clin Pharmacol. 2012;73:812-820.

48. Bauer TK, Lindenbaum K, Stroka MA, Engel S, Linder R, Verheyen F. Fall risk increasing drugs and injuries of the frail elderly evidence from administrative data. Pharmacoepidemiol Drug Saf. 2012;21:1321-1327.

49. Modén B, Merlo J, Ohlsson H, Rosvall M. Psychotropic drugs and falling accidents among the elderly: a nested case control study in the whole population of Scania, Sweden. J Epidemiol Community Health. 2010;64:440-446. 
50. Mehta S, Chen H, Johnson ML, Aparasu RR. Risk of falls and fractures in older adults using antipsychotic agents: a propensity-matched retrospective cohort study. Drugs Aging. 2010;27:815-829.

51. Sorock GS, Quigley PA, Rutledge MK, et al. Central nervous system medication changes and falls in nursing home residents. Geriatr Nurs. 2009;30:334-340.

52. Payne RA, Abel GA, Simpson CR, Maxwell SR. Association between prescribing of cardiovascular and psychotropic medications and hospital admission for falls or fractures. Drugs Aging. 2013;30:247-254.

53. Echt MA, Samelson EJ, Hannan MT, Dufour AB, Berry SD. Psychotropic drug initiation or increased dosage and the acute risk of falls: a prospective cohort study of nursing home residents. $B M C$ Geriatr. 2013;13:19.

54. Chan $\mathrm{CH}$, Gau SS, Chan HY, et al. Risk factors for falling in psychiatric inpatients: a prospective, matched case-control study. J Psychiatr Res. 2013;47:1088-1094.

55. Berry SD, Zhang Y, Lipsitz LA, Mittleman MA, Solomon DH, Kiel DP. Antidepressant prescriptions: an acute window for falls in the nursing home. J Gerontol A Biol Sci Med Sci. 2011;66:1124-1130.

56. Butt DA, Mamdani M, Austin PC, Tu K, Gomes T, Glazier RH. The risk of falls on initiation of antihypertensive drugs in the elderly. Osteoporos Int. 2013;24:2649-2657.

57. Berdot S, Bertrand M, Dartigues JF, et al. Inappropriate medication use and risk of falls - a prospective study in a large community-dwelling elderly cohort. BMC Geriatr. 2009;9:30.

58. Dalleur O, Spinewine A, Henrard S, Losseau C, Speybroeck N, Boland B. Inappropriate prescribing and related hospital admissions in frail older persons according to the STOPP and START criteria. Drugs Aging. 2012;29:829-837.

59. Agashivala N, Wu WK. Effects of potentially inappropriate psychoactive medications on falls in US nursing home residents: analysis of the 2004 National Nursing Home Survey database. Drugs Aging. 2009;26:853-860

60. Beer C, Hyde Z, Almeida OP, et al. Quality use of medicines and health outcomes among a cohort of community dwelling older men: an observational study. Br J Clin Pharmacol. 2011;71:592-599.

61. McMahon CG, Cahir CA, Kenny RA, Bennett K. Inappropriate prescribing in older fallers presenting to an Irish emergency department. Age Ageing. 2014;43:44-50.

62. Berry SD, Quach L, Procter-Gray E, et al. Poor adherence to medications may be associated with falls. J Gerontol A Biol Sci Med Sci. 2010;65:553-558.
63. Ruiz MA, Reske T, Cefalu C, Estrada J. Falls in HIV-infected patients: a geriatric syndrome in a susceptible population. J Int Assoc Provid AIDS Care. 2013;12:266-269.

64. van der Velde N, Meerding WJ, Looman CW, Pols HA, van der Cammen TJ. Cost effectiveness of withdrawal of fall-risk-increasing drugs in geriatric outpatients. Drugs Aging. 2008;25:521-529.

65. van der Velde N, Stricker BH, Pols HA, van der Cammen TJ. Risk of falls after withdrawal of fall-risk-increasing drugs: a prospective cohort study. Br J Clin Pharmacol. 2007;63:232-237.

66. Joester J, Vogler CM, Chang K, Hilmer SN. Hypnosedative use and predictors of successful withdrawal in new patients attending a falls clinic: a retrospective, cohort study. Drugs Aging. 2010;27:915-924.

67. Kelly DM, Frick EM, Hale LS. How the medication review can help to reduce risk of falls in older patients. JAAPA. 2011;24:30-34, 55.

68. Zermansky AG, Alldred DP, Petty DR, et al. Clinical medication review by a pharmacist of elderly people living in care homes - randomised controlled trial. Age Ageing. 2006;35:586-591.

69. Ferreri S, Roth MT, Casteel C, Demby KB, Blalock SJ. Methodology of an ongoing, randomized controlled trial to prevent falls through enhanced pharmaceutical care. Am J Geriatr Pharmacother. 2008;6:61-81.

70. Blalock SJ, Casteel C, Roth MT, Ferreri S, Demby KB, Shankar V. Impact of enhanced pharmacologic care on the prevention of falls: a randomized controlled trial. Am J Geriatr Pharmacother. 2010;8:428-440.

71. Haumschild MJ, Karfonta TL, Haumschild MS, Phillips SE. Clinical and economic outcomes of a fall-focused pharmaceutical intervention program. Am J Health Syst Pharm. 2003;60:1029-1032.

72. Tamblyn R, Eguale T, Buckeridge DL, et al. The effectiveness of a new generation of computerized drug alerts in reducing the risk of injury from drug side effects: a cluster randomized trial. J Am Med Inform Assoc. 2012;19:635-643.

73. Tzeng HM, Hu HM, Yin CY. Is there a link between the hospital-acquired injurious fall rates in US acute care hospitals and these institutions' implementation levels of computerized systems? Comput Inform Nurs. 2011;29:721-729.

74. Sato Y, Iwamoto J, Honda Y, Amano N. Vitamin D reduces falls and hip fractures in vascular parkinsonism but not in Parkinson's disease. Ther Clin Risk Manag. 2013;9:171-176.

75. Lan MJ, Zhu LL, Zhou Q. Medication administration errors made by nurses reflect the level of pharmacy administration and hospital information infrastructure. J Clin Nurs. 2014;23:894-895.
Therapeutics and Clinical Risk Management

\section{Publish your work in this journal}

Therapeutics and Clinical Risk Management is an international, peerreviewed journal of clinical therapeutics and risk management, focusing on concise rapid reporting of clinical studies in all therapeutic areas, outcomes, safety, and programs for the effective, safe, and sustained use of medicines. This journal is indexed on PubMed Central, CAS,
Dovepress

EMBase, Scopus and the Elsevier Bibliographic databases. The manuscript management system is completely online and includes a very quick and fair peer-review system, which is all easy to use. Visit http://www.dovepress.com/testimonials.php to read real quotes from published authors. 